\section{BRAZIULIAN JOURNAL}

OF MEDICAL AND BIOLOGICAL RESHARCH

www.bjournal.com.br
ISSN 0100-879X

Volume 42 (8) 692-775 August 2009

CLINICAL INVESTIGATION

Braz J Med Biol Res, August 2009, Volume 42(8) 692-699

Methodological approaches to planar and volumetric scintigraphic imaging of small volume targets with high spatial resolution and sensitivity

J. Mejia, O.Y. Galvis-Alonso, J. Braga, R. Corrêa, J.P. Leite and M.V. Simões

The Brazilian Journal of Medical and Biological Research is partially financed by
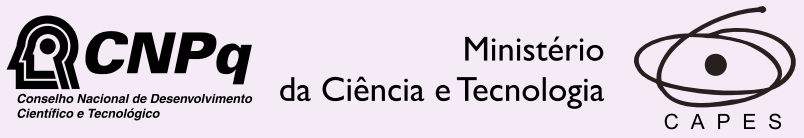

Ministério da Educação

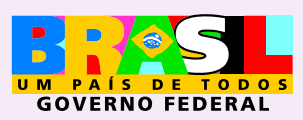

Institutional Sponsors 


\title{
Methodological approaches to planar and volumetric scintigraphic imaging of small volume targets with high spatial resolution and sensitivity
}

\author{
J. Mejia ${ }^{1}$, O.Y. Galvis-Alonso ${ }^{1}$, J. Braga², R. Corrêa ${ }^{3}$, \\ J.P. Leite ${ }^{4}$ and M.V. Simões ${ }^{5}$ \\ ${ }^{1}$ Departamento de Biologia Molecular, Faculdade de Medicina de São José do Rio Preto, \\ São José do Rio Preto, SP, Brasil ${ }^{2}$ Divisão de Astrofísica, ${ }^{3}$ Ciência Espacial e Atmosférica, \\ Instituto Nacional de Pesquisas Espaciais, São José dos Campos, SP, Brasil \\ ${ }^{4}$ Departamento de Neurologia, Psiquiatria e Psicologia Médica, ${ }^{5}$ Departamento de Clínica Médica, \\ Faculdade de Medicina de Ribeirão Preto, Universidade de São Paulo, Ribeirão Preto, SP, Brasil
}

\begin{abstract}
Single-photon emission computed tomography (SPECT) is a non-invasive imaging technique, which provides information reporting the functional states of tissues. SPECT imaging has been used as a diagnostic tool in several human disorders and can be used in animal models of diseases for physiopathological, genomic and drug discovery studies. However, most of the experimental models used in research involve rodents, which are at least one order of magnitude smaller in linear dimensions than man. Consequently, images of targets obtained with conventional gamma-cameras and collimators have poor spatial resolution and statistical quality. We review the methodological approaches developed in recent years in order to obtain images of small targets with good spatial resolution and sensitivity. Multipinhole, coded mask- and slit-based collimators are presented as alternative approaches to improve image quality. In combination with appropriate decoding algorithms, these collimators permit a significant reduction of the time needed to register the projections used to make 3-D representations of the volumetric distribution of target's radiotracers. Simultaneously, they can be used to minimize artifacts and blurring arising when single pinhole collimators are used. Representation images are presented, which illustrate the use of these collimators. We also comment on the use of coded masks to attain tomographic resolution with a single projection, as discussed by some investigators since their introduction to obtain near-field images. We conclude this review by showing that the use of appropriate hardware and software tools adapted to conventional gamma-cameras can be of great help in obtaining relevant functional information in experiments using small animals.
\end{abstract}

Key words: Small animal SPECT; Multipinhole collimators; Coded masks

\section{Introduction}

In the last few decades, and mainly since the development of genetically modified animals, interest in animal models of human diseases has grown, especially with the use of rats and mice. Animal models are necessary in areas such as genetics, physiology, physiopathology and, mainly, for the discovery and testing of new pharmaceuticals. Small animals such as rats and mice are particularly important because of their easy reproduction, maintenance in small spaces and the knowledge available about their genetic background (e.g., Ref. 1).

Noninvasive imaging techniques are very important tools in this kind of experiments since they permit sequential recording for the same animal in vivo (2). Therefore, the animal under study can be used as its own control, making it easier to interpret results and, at the same time, reducing the number of animals necessary for an experiment as well as research time and costs. A subset of these imaging techniques is Nuclear Medicine imaging, which encompasses

Correspondence: J. Mejia, Departamento de Biologia Molecular, Faculdade de Medicina de São José do Rio Preto, Av. Brigadeiro Faria Lima, 5416, 15090-000 São José do Rio Preto, SP, Brasil. E-mail: mejia_famerp@yahoo.com.br

J. Mejia is supported by FAPESP (\#07/50339-3 and \#07/58052-5). J.P. Leite is supported by FAPESP CInAPCe (\#05/56447-7). Received April 9, 2009. Accepted June 26, 2009. 
positron emission tomography (PET) and single-photon emission computed tomography (SPECT).

SPECT and PET are imaging techniques based on the detection of high-energy photons emitted from the target after the introduction of a radioactive nuclide incorporated into a radio-probe and targeting specific tissues. In the case of PET, the radionuclide emits a positron, which travels a short distance in the target until finding an electron. Their annihilation produces two $511-\mathrm{keV}$ photons traveling in almost opposite directions. In SPECT, individual noncorrelated high-energy photons are emitted and detected. In both cases, SPECT and PET, emission of photons occurs at all angles around the emitting center, giving rise to a spherical distribution of radiation. Compared with PET, radionuclides and radiopharmaceuticals appropriate for SPECT are more easily available (e.g., ${ }^{18} \mathrm{~F},{ }^{15} \mathrm{O}$ and ${ }^{11} \mathrm{C}$ for PET versus ${ }^{99 \mathrm{~m} T c},{ }^{111} \mathrm{In},{ }^{123} \mathrm{I},{ }^{125} \mathrm{I},{ }^{67} \mathrm{Ga}$, and ${ }^{201} \mathrm{Ta}$ for SPECT), with longer mean lives than their PET counterparts, apart from needing less expensive technology in their production. Additionally, SPECT radionuclides emit in different energies, which allow, at least in principle, simultaneous dual-isotope recording. On the other hand, the typical sensitivity and resolution in clinical assays are better for PET than for SPECT, other parameters being the same (3). Despite its disadvantages, SPECT is a more common used imaging tool in hospitals.

In order to produce SPECT images, the incidence direction of the photons on the detector must be codified in some way. In the most general cases, the codification is done by the use of single pinhole or parallel-hole collimators to produce planar projections. Planar images, acquired from sequential angles around the target, are combined to produce 3-D reconstructions. The techniques used to produce the 3-D reconstructions include various versions of the filtered backprojection algorithm (3), maximum likelihood iterative restoration algorithms (see, e.g., Ref. 4) and, more recently, the faster ordered subset + expectation maximization algorithm (5). Gamma cameras based on scintillation crystals [e.g., $\mathrm{Nal}(\mathrm{TI})$ ] are the most common type of cameras currently in use. They are limited in spatial and spectral resolution to $\sim 4 \mathrm{~mm}$ (intrinsic detector resolution) on the image plane and 10\% @ 140 keV, respectively (see, e.g., Ref. 3), imposing limitations on the quality of final images. The maximum radiation dose to be applied to patients places an additional limit to the standard minimum hole diameter in pinhole collimators of roughly $4 \mathrm{~mm}$ to reach acceptable statistical significance, which in turn sets a limit on the spatial resolution on the object plane. The maximum dose also imposes a limit on dynamic studies.

In the last two decades, several groups have been working on developing techniques or adapting the available ones to obtain Nuclear Medicine images of small animals. In the present study, we describe different alternatives developed to improve spatial resolution and sensitivity when making SPECT images of small size targets. In the following section, we describe the use of multipinhole collimators in combination with iterative image restoration algorithms, and compare its performance with that of single pinhole and parallel hole collimators. In the section entitled "Scintigraphic imaging with coded mask-based collimators", we present the coded mask technique as applied in the astrophysics field, how it can be applied to near field imaging and the advantages it presents over other imaging alternatives. In the section entitled "Slit-based collimators", we comment on the use of non-standard collimators based on slits, such as the simple slit, slit-slat and skew slit collimators. In the section entitled "Three-dimensional reconstructions from planar projections", the application to 3-D reconstructions of volumetric targets is considered. Finally, the conclusions of the present study are summarized in the last paragraph of this article.

\section{Scintigraphic imaging with multipinhole collimators}

When single pinhole or parallel hole collimators are used to obtain scintigraphic images, spatial resolution is limited by the combination of the detector's intrinsic resolution and the smearing effect due to the physical size of the collimator's holes, as indicated by the expressions

$R_{o} \cong \sqrt{\left[\left(1+\frac{d_{o}}{d_{i}}\right) d_{\text {eff }}\right]^{2}+\left[R_{i} \frac{d_{o}}{d_{i}}\right]^{2}}$

for pinhole collimators and

$R_{o} \cong \sqrt{\left[\left(1+\frac{d_{o}}{h}\right) d_{e f f}\right]^{2}+R_{i}^{2}}$

for parallel hole collimators,

where $d_{o}$ and $d_{i}$ are the distances from the collimator to the object and to the detector, respectively, $h$ is the height of the parallel hole collimator, $d_{\text {eff }}$ is the effective diameter of the collimator considering partial transparency of the collimator's material, and $R_{i}$ is the intrinsic resolution of the detector. Because of the magnification factor intrinsic to pinhole collimators (given by the $d_{i} / d_{0}$ ratio), the effect of the detector's intrinsic resolution on the final quality of the image is less pronounced in this technique, even though a reduction in the field of view (FOV) is implicit. Small diameter pinhole collimators are, then, desirable when imaging small size objects. However, reducing the open area in the aperture implies a quadratic reduction of sensitivity, making it necessary to increase the exposure time or the dose applied to attain an equivalent statistical significance of the image.

To overcome this conflicting situation, the use of multiple small-sized pinholes in the collimator has been proposed 
with two different alternatives: a) multipinhole collimators, where roughly a dozen pinholes are used to record the target's activity (see, Refs. 6,7) and b) coded mask collimators, where a higher number of pinholes (dozens to hundreds of holes) following a distribution with special mathematical properties are used (8). Examples of both kinds of collimators are shown in Figure 1. In both cases, the recorded image is no longer a direct representation of the target, but results from the superposition of the individual images projected by each pinhole, which can partially overlap depending on parameters such as object size, distance between pinholes, and magnification. It is said that the target's representation is coded and a second decoding stage is necessary.

In the most general way, the recorded image can be represented by the convolution of the intensity distribution on the object plane with the collimator pattern, as given by the expression

$S=A \otimes O+N$,

[3]

where $S$ represents the recorded image (sometimes called shadowgram), $O$ corresponds to the intensity distribution on the object plane, $A$ is the aperture array, $N$ corresponds to a noise term, and $\otimes$ stands for the convolution product. Thus, the objective is to decode the recorded image in order to obtain a representation of the target.

This decoding phase is usually carried out with iterative image restoration algorithms, such as the maximum likelihood algorithm (see Ref. 9). Image restoration can be understood as finding the reproducible object intensity distribution, which better explains the detected intensity

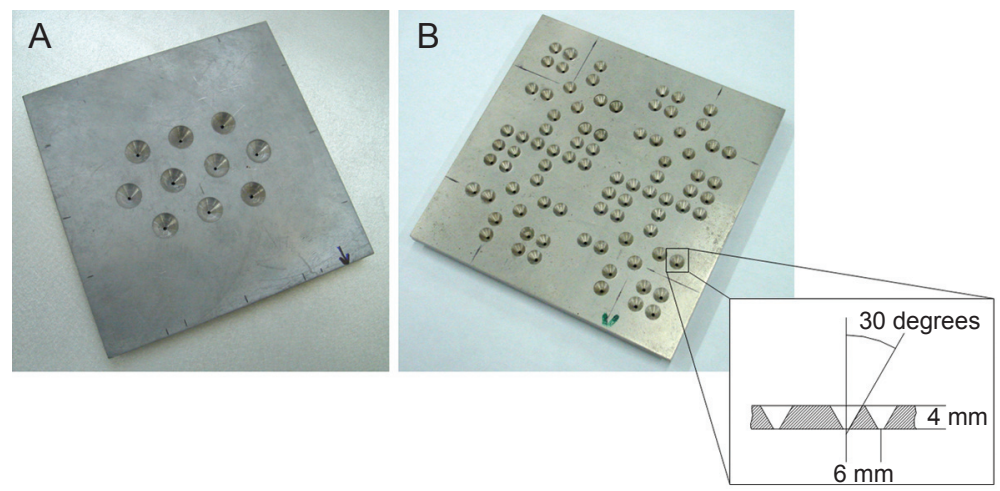

Figure 1. Multipinhole (A) and a coded mask-based (B) collimator with details of the pinhole profile. Both collimators were constructed on 4-mm thick lead plates, which guarantee transparency of less than $0.1 \%$ at $140 \mathrm{keV}$. Distances between holes and total aperture angle are 15 and $6 \mathrm{~mm}$, and 90 and 60 degrees, respectively. distribution, given the instrument's characteristics and the irreproducible noise contribution. This kind of problem is known as the inverse problem and arises in many scientific areas. Following Equation [3], the objective is to find a model of the object intensity distribution, $O(x)$, given the detected intensity distribution, $S(x)$, and the response of the instrument to a point source, $A(x)$.

According to this kind of algorithms, a first model of the object intensity distribution is proposed, typically a uniform one. This distribution is projected through the instrument's model to obtain a recorded image, which is compared to the real data. Correction factors are determined and back-projected through the instrument to the object space in order to be applied to the original object's model. The entire process is repeated with the new object distribution, until some finishing criterion is reached. Mathematically, the decoded image can be represented by the recursive expression

\section{Scintigraphic imaging with coded mask-based collimators}

Coded masks have been extensively used for decades in high-energy astrophysics (for reviews, see Refs. 10,11), in order to image astrophysical fields in the energy range where focusing techniques such as grazing incidence are not suitable, i.e., more than $\sim$ tens of keV and up to some MeV. In the coded mask approach, the instrument's aperture consists of a distribution of small size holes on an otherwise opaque screen, following an appropriate pattern. As with multipinhole collimators, every pinhole produces an image of the target onto the detector. However, the positions of the pinholes on the mask are such that the distribution of images on the detector is unique for each point source position in the FOV. As with multipinhole collimators, the information on the photon's incidence direction is encoded in the recorded image, which can be recovered in a second step of image 
decoding by appropriate mathematical manipulations (see, Refs. 8,12). Because of the high number of small size holes on the aperture, coded masks provide a substantial gain in detection efficiency while maintaining the spatial resolution of single pinhole cameras with the same hole size.

In the far-field situation, as is the case of imaging astrophysical sources located at infinity, one of the most efficient methods to decode the image of the observed FOV is the correlation method $(8,12)$. According to it, there exists a decoding array, $G$, such that the correlation product between it and the mask pattern $A, A^{*} G$, is close or equal to a delta-function (a pulse on an otherwise flat or null background). On this basis,

$$
I=S * G=[A \otimes O] * G+N * G=[A * G] \otimes O+N * G=\delta \otimes O+N * G,
$$

the decoded image, $I$, is equivalent to the observed object except for a noise term.

Since the introduction of random hole distributions independently proposed by Dicke (13) and Ables (14), many different classes of coded mask patterns have been discussed in the literature. A special case is that of the uniformly redundant arrays introduced by Fenimore and Cannon (8), a set of pinhole distributions whose autocorrelation function corresponds to a perfect delta-function superimposed on a constant or null level. Additionally, the decoding process is such that the contribution of any uniformly distributed noise pattern is automatically removed.
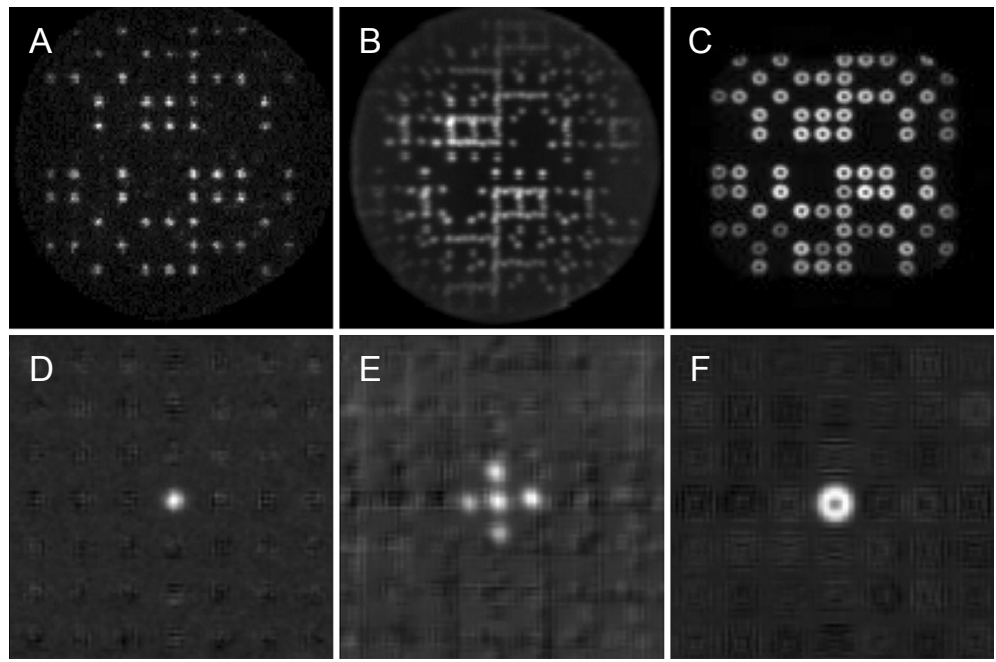

Figure 2. Shadowgrams of a point-like source (A), a set of five point-like sources in a "+" format (B) and a 3-mm diameter ring (C), as well as the corresponding decoded images (D, E, F). The images were obtained with a Siemens-Orbiter gamma-camera in combination with a $7 \times 7$-MURA coded mask collimator, and pixellated on $128 \times 128$ element arrays. Decoding was done using the correlation method. Objects were located $50 \mathrm{~mm}$ from the collimator and the magnification factor was 4 . For the point-like source, a resolution of $3.55 \mathrm{~mm}$ (full-width at halfmaximum) was obtained.
A spatially non-uniform background noise pattern can be removed by the use of the mask-antimask technique (15). In this approach, two shadowgrams are registered, one through the mask pattern and the other through the negative of the previous mask pattern. Subtraction of the second record from the first one before image decoding removes the background contribution, provided the background intensity is constant with time.

Coded masks were proposed as an alternative to produce planar images of objects in the near field by Cannon and Fenimore (16), Fenimore et al. (17), and Chen et al. (18). In addition, these investigators showed that these kinds of collimators present some tomographic depth resolution, which allows the identification of individual planes a couple of centimeters away from each other. They were also used to produce tomographic images of phantoms and biological specimens by, e.g., Rogers et al. (19), who obtained images of a dog model of acute myocardial infarction.

The use of coded masks in near-field imaging was reappraised by Accorsi et al. (20) who discussed the most appropriate coded mask patterns in terms of signal-to-noise ratio (21), and indicated the modified uniformly redundant arrays (22) as an appropriate option for this application. The origin of artifacts appearing in this application was also reviewed (23), mainly related to the fact of partial coding of the FOV, and the mask-antimask technique (15) was suggested as an alternative to remove them by hardware, not requiring additional time or computation effort to produce an image. Additionally, they present images of small FOVs produced by combining a coded mask-based collimator with conventional Anger cameras. When decoded by the classical correlation method, high spatial $(\sim 1.7$ $\mathrm{mm})(20)$ and temporal ( $50 \mathrm{~ms} /$ frame with weak sources) (24) resolution planar images were obtained, indicating that the technique can be used for dynamic studies of radiotracers. Examples of planar images of different targets can be seen in Figure 2. In this figure we show the recorded and decoded images corresponding to a pointlike source, a set of five point-like sources in a "+" format and $5 \mathrm{~mm}$ from one another, and a 3-mm diameter ring. Those images were produced using the $7 \times 7$ MURAbased coded mask collimator, cyclically extended to $13 \times 13$ elements, presented in Figure 1B. The correlation method was used to obtain the decoded images.

Alternatively, the same recorded images can be decoded by means of iterative restoration image algorithms, as described previously. In this case, the coded mask collimator is considered as a multipinhole collimator, not necessarily taking 
advantage of the correlation properties associated with the pinhole distribution during the decoding process. This approach was considered by Mu and Liu (25), who found that it allows removal of most of the noise artifacts in the restored image. In Figure 3, we show the decoded images corresponding to the targets presented in Figure 2 by using iterative restoration algorithms. It can be seen that, when considering small targets, restored images present a more uniform and almost absent background if compared to the results obtained with the correlation decoding algorithm.

Both kinds of collimators discussed here previously, as well as the decoding techniques, can be easily adapted to obtain simple projections of plane objects (objects whose dimension in depth is much smaller than transverse dimensions). When imaging volumetric objects, single decoded projections are not so directly obtained. In this case all the images of the individual planes, projected on the detector for each pinhole and subject to different magnification factors, are superimposed, hindering an appropriate reconstruction. In other words, as a depth source is moved from the axis of a pinhole collimator to the border of its FOV, its projection on the detector changes. This situation, added to the fact that the same effect happens with each pinhole at different positions, causes the recorded image to become blurred and prevents the restoration of single planar projections.

Since the earliest application of coded masks to near field imaging, it has been argued that this kind of collimator provides a way to identify individual depth planes in the target $(16-19,25,26)$. This property is thought to be due to the fact that sources on different depth planes project mask shadows of different sizes on the detector. At least in principle, individual planes can be independently identified if the decoding array is appropriately magnified in the same proportion as the shadow of the mask pattern, as produced by a point source on those planes. This argument would be valid if the cross-correlation between the shadow of the mask and the different magnified projections of the decoding array were a delta-function. Partial correlation exists between them, which introduces artifacts and hampers an appropriate reconstruction. However, under appropriate conditions, individual planes can be identified. This is the case if the signal in the target is sparse, consisting of discrete sources located on relatively distant planes. Mu and Liu (25) have shown that, by decoding iteratively, some tomographic depth from a single projection can be recovered. In Figure 4 , we show the case of an object consisting of two parallel lines separated by $10 \mathrm{~mm}$ at
$50 \mathrm{~mm}$ from the collimator and a 10-mm diameter ring, 10 $\mathrm{mm}$ away. Even though individual planes can be recovered, some cross-talk is still evident. The quality of the decoded images depends on several factors such as the distance between individual source planes, the number of intermediate planes considered in the decoding process and the relative intensity and size of the sources.

\section{Slit-based collimators}

As indicated in the Section entitled "Scintigraphic imaging with multipinhole collimators", when pinhole collimators are used to produce high spatial resolution images, the FOV becomes limited, its size depending on the magnification factor. Objects at the border of the pinhole's FOV project onto the detector at an angle different from the one corresponding to objects near the center. In this way, the borders of the recorded image become blurred. To overcome this limitation, it has been proposed to use long collimators in the format of slits. This is the case for the slit-slat (27-29) and skew-slit (30) collimators, which, additionally, allow for an increase in sensitivity while maintaining high spatial
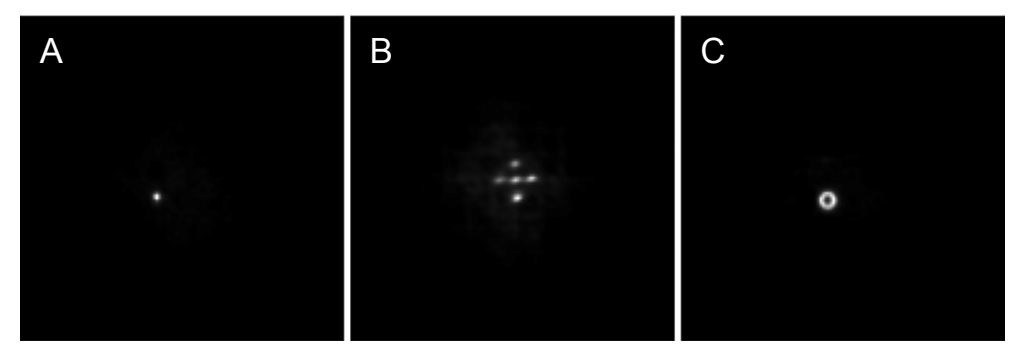

Figure 3. Decoded images (A, B, C) corresponding to the same objects used to obtain the records shown in Figure 2 (A, B, C), respectively. Decoding was done with an iterative maximum likelihood-based restoration algorithm, with 15 iterations. Note how the background is greatly reduced when compared with the decoded images shown previously (Figure $2 \mathrm{D}, \mathrm{E}, \mathrm{F}$ ). For the point-like source, a resolution of $1.66 \mathrm{~mm}$ (full-width at half-maximum) was obtained.
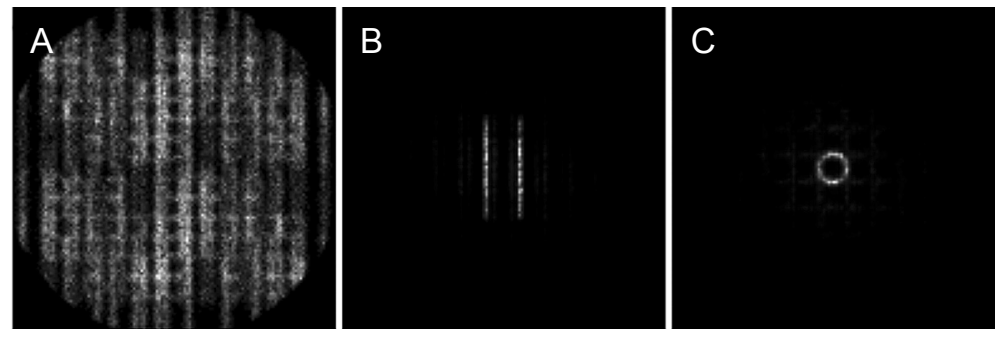

Figure 4. $A$, Simulated shadowgram projected on the detector by a set of two linear sources $10 \mathrm{~mm}$ from each other, located $50 \mathrm{~mm}$ from the detector, with a 10 -mm outer diameter, 7-mm inner diameter ring located $60 \mathrm{~mm}$ from the collimator. $B$, Decoded image of the plane at $50 \mathrm{~mm}$ and $C$, at $60 \mathrm{~mm}$. The presence of 21 intermediate planes, 45 to $65 \mathrm{~mm}$ from the collimator, was considered while image decoding. 
resolution. In the first case, the slit and slats are mutually perpendicular. Slats behave as parallel collimators, which causes axial slices to be independent from one another, while the slit behaves as a continuous sequence of pinholes in the perpendicular (or transaxial) direction. In the second case, the slits are located at different distances from the detector, which produces different and independent magnifications for each direction. As with slit-slat collimators, a skew-slit guarantees high spatial resolution in one of the directions and an extension of the FOV in the other, thus reducing artifacts.

A single slit was also proposed, in combination with a linear detector (31), as an alternative to reach high spatial resolution if compared with single pinhole collimators. In this application, a set of cadmium zinc telluride elements is mounted in a linear format instead of a rectangular one and is allowed to spin around the optical axis while rotating around the target, scanning a larger detection area. However, because of the relative position of the slit perpendicular to the rotating direction, this system is still limited in FOV and displays artifacts in the axial direction.

\section{Three-dimensional reconstructions from planar projections}

Multipinhole and slit-based collimators have been used to obtain 3-D images of volumetric distributions of radiotracer (SPECT). In order to obtain individual projections necessary to make $3-D$ reconstructions, the target is rotated around its own axis, instead of the heavy gamma-camera head, thus minimizing misalignment errors. Positioning can be guaranteed by step-motor-based computer-controlled rotation systems (see Refs. 32-34). Alternatively, the target is maintained stationary and the hole pattern is drilled in a tube, which completely surrounds the target. The collimator and the camera head, without the now unnecessary shielding, are rotated around the object of study (35). In other applications, a set of detectors is mounted completely surrounding the target, as is the case, for example, for U-SPECT (36) and fast-SPECT (37), while the complete system is maintained stationary.

Even though individual projections made with multipinhole or slit-based collimators are not easily decoded, their combined information content can be sufficient to produce an appropriate representation of the volumetric distribution of radiotracer in the target. Extensions to three dimensions of the iterative algorithms like the one described in the section entitled "Scintigraphic imaging with multipinhole collimators" have been used in these applications. As the quantity of information grows with the number of projections acquired, the time span for each decoding iteration is also increased. An acceleration procedure, which has given good results, is known as ordered subset - expectation maximization (5). In this approach, different subsets of projections are used in each iteration. Depending on the number of projections in each subset, the total analysis time can be greatly reduced.

On the other hand, if the pinholes are located along a direction perpendicular to the axis of rotation, individual images projected on the detector by each one of them are equivalent to projections taken by a single pinhole collimator from a rotation angle equal to the angle subtended by the displaced pinhole. Depending on the magnification factor and the detector's size, several projections are recorded simultaneously. In this way, the number of total intermediate positions is reduced, as well as the decoding time per iteration.

In Figure 5A, we show a set of 20 projections of a curved catheter $1 \mathrm{~mm}$ in internal diameter, acquired with a collimator consisting of $41-\mathrm{mm}$ diameter pinholes. The catheter was filled with a small quantity (less than $0.1 \mathrm{mCi}$ ) of $99 \mathrm{~m} \mathrm{Tc}$. The projections were registered on a matrix of $128 \times 128$ elements, $3.5 \mathrm{~mm} /$ pixel, with a DST-Sopha gamma camera (Sopha Medical Vision, USA/France), with an integration time of 30 s/iteration. The center of rotation was located $50 \mathrm{~mm}$ away from the collimator, which was located 200 $\mathrm{mm}$ from the detector surface. The distance between the branches of the catheter was $5 \mathrm{~mm}$ at the center of the object. Transverse and horizontal planes through the center of the decoded image are shown in Figure 5B. Decoding was done with an implementation of the maximum likelihood algorithm, with a total of 5 iterations.

To summarize, in the present review we report details of the methodological approaches that have been proposed to obtain high-quality planar and volumetric scintigraphic images of small volume targets. Pinhole collimators are the obvious option for this application because of the implicit magnification factor. By using most of the detector's area to project an image of the small size target, the limiting effect of the detector's intrinsic resolution is minimized. Additionally, by using small diameter holes, the photon's direction of incidence is better determined and an additional improvement in spatial resolution is achieved. However, sensitivity is greatly reduced. Multipinhole and coded mask-based collimators, in combination with appropriate decoding algorithms, have been used to reach simultaneously high spatial resolution and sensitivity. Depending on the distribution of the pinholes in the collimators, the total recording time necessary to make a volumetric 3-D representation of the target can be reduced by registering several projections simultaneously. If the number of individual projections or of the pinholes in the collimators increases, the decoding time also grows. The iterative decoding procedure can be accelerated by algorithms like ordered subset + expectation maximization, where a subset of the projections is considered in each individual iteration. Slit-based collimators have also been considered. They have been used as single slits, and in combination with a second one or with slats, both perpendicular to the first slit. This kind of collimator guarantees high spatial 

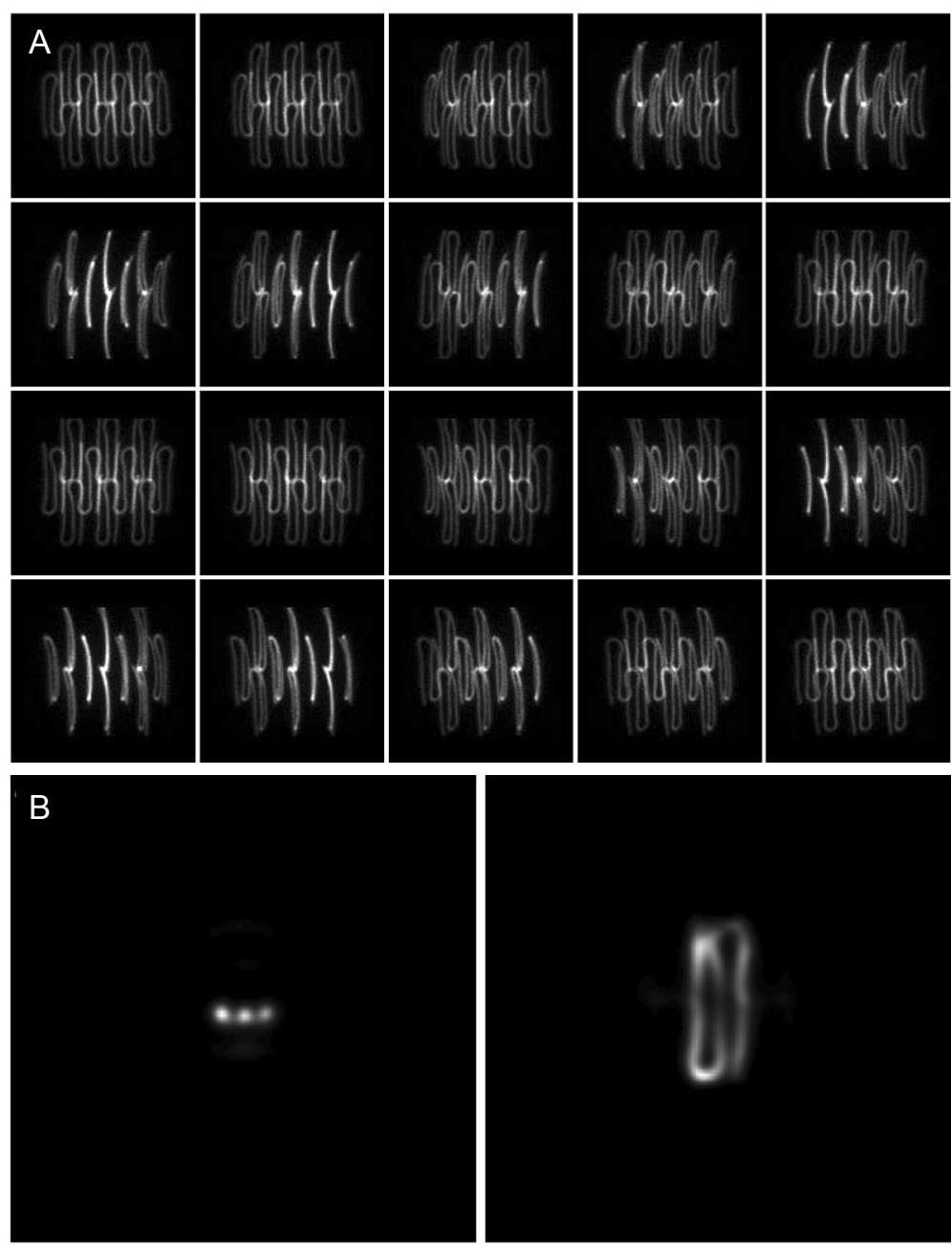

Figure 5. A, Twenty projections of a curved 1-mm diameter catheter filled with $<0.1 \mathrm{mCi}$ of ${ }^{99 \mathrm{mTc}}$, acquired with a multipinhole collimator consisting of $10 \mathrm{pin}$ holes $1 \mathrm{~mm}$ in diameter, as shown in Figure 1A. B. Transverse and horizontal planes through the center of the decoded object. Decoding was done with an iterative algorithm based on maximum likelihood expectation maximization, with 5 iterations. resolution in at least one dimension, while displaying high sensitivity. Additionally, the slit provides a better sampling of the target, reducing decoding artifacts normally associated with single pinhole collimators. It has been argued that coded mask-based collimators can be used to obtain tomographic resolution with a single image. Even though this is true under some special conditions, the ability to resolve individual depth planes depends on several parameters such as the intensity distribution in the target, the distance between individual source elements, and the number and thickness of the intermediate planes the object is considered to consist of. Finally, good quality planar and volumetric scintigraphic images of small FOV can be obtained with the technology and equipment available in medical institutions, by adapting clinical gamma-cameras, in combination with low cost special purpose collimators and the appropriate processing software tools. The application of these approaches can allow us to obtain better functional images in biomedical research, which can be achieved in clinical studies of small volume body parts in clinical or in experimental studies using small animals.

\section{Acknowledgments}

We wish to thank the technical staff of the Nuclear Medicine Section, Hospital das Clínicas, Universidade de São Paulo, Ribeirão Preto, SP, Brazil, for invaluable help in preparing and manipulating the phantoms during the tests.

\section{References}

1. Institute of Laboratory Animal Resources (U.S.). Committee on rodents, laboratory animal management: rodents. Washington: National Academy Press; 1996.

2. Dzik-Jurasz AS. Molecular imaging in vivo: an introduction. Br J Radiol 2003; 76 (Spec No. 2): S98-S109.

3. Cherry SR, Sorenson JA, Phelps ME. Physics in nuclear medicine. 3rd edn. Philadelphia: Elsevier Science; 2003.

4. Shepp LA, Vardi Y. Maximum likelihood reconstruction for emission tomography. IEEE Trans Med Imaging 1982; 1: 113-122.

5. Hudson HM, Larkin RS. Accelerated image reconstruction using ordered subsets of projection data. IEEE Trans Med Imaging 1994; 13: 601-609.
6. Meikle SR, Kench P, Weisenberger AG, Wojcik R, Smith MF, Majewski $S$, et al. A prototype coded aperture detector for small animal SPECT. IEEE Trans Nucl Sci 2002; 49: 21672171.

7. Schramm UN, Schipper M, Schurrat T, Behe M, Alfke H, Engeland $U$, et al. Performance of a multi-pinhole animal SPECT. IEEE Nucl Sci Symp Conf Rec 2003; 3: 20772079.

8. Fenimore EE, Cannon TM. Coded aperture imaging with uniformly redundant arrays. Appl Opt 1978; 17: 337-347.

9. Puetter RC, Gosnell TR, Yahil A. Digital image reconstruction: Deblurring and denoising. Annu Rev Astron Astrophys 2005; 43: 139-194. 
10. Skinner GK. Imaging with coded-aperture masks. Nucl Instrum Methods Phys Res A 1984; 221: 33-40.

11. Caroli E, Stephen JB, diCocco G, Natalucci L, Spizzichino A. Coded-aperture imaging in X-and gamma-ray astronomy. Space Sci Rev 1987; 45: 349-403.

12. Fenimore EE, Cannon TM. Uniformly redundant arrays: digital reconstruction methods. Appl Opt 1981; 20: 1858-1864.

13. Dicke RH. Scatter-hole cameras for X-rays and gammarays. Astrophys J 1968; 153: L101-L106.

14. Ables JG. Fourier transform photography: a new method for X-ray astronomy. Proc ASA 1968; 1: 172-173.

15. Jayanthi UB, Braga J. Physical implementation of an antimask in URA based coded mask systems. Nucl Instrum Methods Phys Res A 1991; 310: 685-689.

16. Cannon T, Fenimore EE. Tomographical imaging using uniformly redundant arrays. Appl Opt 1979; 18: 1052-1057.

17. Fenimore EE, Cannon TM, Van Hulsteyn DB, Lee P. Uniformly redundant array imaging of laser driven compressions: preliminary results. Appl Opt 1979; 18: 945-947.

18. Chen Y-W, Yamanaka M, Miyanaga N, Yamanaka T, Nakai $\mathrm{S}$, Yamanaka $\mathrm{C}$, et al. Three-dimensional reconstructions of laser-irradiated targets using URA coded aperture cameras. Opt Commun 1989; 71: 249-255.

19. Rogers WL, Koral KF, Mayans R, Leonard PF, Thrall JH, Brady TJ, et al. Coded-aperture imaging of the heart. $J$ Nucl Med 1980; 21: 371-378 (Abstract).

20. Accorsi R, Gasparini F, Lanza RC. A coded aperture for high-resolution nuclear medicine planar imaging with a conventional Anger camera: experimental results. IEEE Trans Nucl Sci 2001; 48: 2411-2417.

21. Accorsi R, Gasparini F, Lanza RC. Optimal coded aperture patterns for improved SNR in nuclear medicine imaging. Nucl Instrum Methods Phys Res A 2001; 474: 273-284.

22. Gottesman SR, Fenimore EE. New family of binary arrays for coded aperture imaging. Appl Opt 1989; 28: 4344-4352.

23. Accorsi R, Lanza RC. Near-field artifact reduction in planar coded aperture imaging. Appl Opt 2001; 40: 4697-4705.

24. Accorsi R, Lanza RC. High-sensitivity dynamic coded aperture imaging. IEEE Nucl Sci Symp Conf Rec 2003; 3: 1833-1837.

25. Mu Z, Liu YH. Aperture collimation correction and maximumlikelihood image reconstruction for near-field coded aperture imaging of single photon emission computerized tomography. IEEE Trans Med Imaging 2006; 25: 701-711.
26. Schellingerhout $D$, Accorsi R, Mahmood U, Idoine J, Lanza RC, Weissleder R. Coded aperture nuclear scintigraphy: a novel small animal imaging technique. Mol Imaging 2002; 1 : 344-353.

27. Metzler SD, Accorsi R, Novak JR, Ayan AS, Jaszczak RJ. On-axis sensitivity and resolution of a slit-slat collimator. $J$ Nucl Med 2006; 47: 1884-1890.

28. Metzler SD, Ayan AS, Accorsi R, Novak JR. Reconstruction of phantom SPECT scans acquired with a slit-slat collimator. IEEE Nucl Sci Symp Conf Rec 2006; M14-27: 2966-2970.

29. Metzler SD, Accorsi R, Ayan AS, Novak JR, Lewitt RM, Jaszczak RJ. Preliminary assessment of multislit-slat collimation for cardiac SPECT. IEEE Nucl Sci Symp Conf Rec 2007; M18-54: 3349.

30. Tang $Q$, Zeng GL, Huang Q. An analytical algorithm for skew-slit imaging geometry with uniform attenuation correction in SPECT. IEEE Nucl Sci Symp Conf Rec 2006; 5: 2822-2825.

31. Zeng GL, Gagnon D. CdZnTe strip detector SPECT imaging with a slit collimator. Phys Med Biol 2004; 49: 2257-2271.

32. Habraken JB, de Bruin K, Shehata M, Booij J, Bennink R, van Eck Smit BL, et al. Evaluation of high-resolution pinhole SPECT using a small rotating animal. J Nucl Med 2001; 42: 1863-1869.

33. Wu MC, Gao DW, Sievers RE, Lee RJ, Hasegawa BH, Dae MW. Pinhole single-photon emission computed tomography for myocardial perfusion imaging of mice. J Am Coll Cardiol 2003; 42: 576-582.

34. Sohlberg A, Lensu S, Jolkkonen J, Tuomisto L, Ruotsalainen $\mathrm{U}$, Kuikka JT. Improving the quality of small animal brain pinhole SPECT imaging by Bayesian reconstruction. Eur $J$ Nucl Med Mol Imaging 2004; 31: 986-994.

35. Difilippo FP. Design and performance of a multi-pinhole collimation device for small animal imaging with clinical SPECT and SPECT-CT scanners. Phys Med Biol 2008; 53: 41854201.

36. Beekman FJ, van der Have F, Vastenhouw B, van der Linden AJ, van Rijk PP, Burbach JP, et al. U-SPECT-I: a novel system for submillimeter-resolution tomography with radiolabeled molecules in mice. J Nucl Med 2005; 46: 1194-1200.

37. Furenlid LR, Wilson DW, Chen Y, Kim H, Pietraski PJ, Crawford MJ, et al. FastSPECT II: a second-generation high-resolution dynamic SPECT imager. IEEE Trans Nucl Sci 2004; 51: 631-635. 